\title{
Antigenic peptide recognition on the human $A B C$ transporter TAP resolved by DNP-enhanced solid-state NMR spectroscopy
}

Elisa Lehnert ${ }^{\dagger, \ddagger}$, Jiafei Mao ${ }^{\|, \ddagger}$, Ahmad Reza Mehdipour ${ }^{\S}$, Gerhard Hummer ${ }^{\S}$, Rupert Abele ${ }^{\dagger}$, Clemens Glaubitz ${ }^{\|, *}$ \& Robert Tampé ${ }^{\dagger, *}$

${ }^{\dagger}$ Institute of Biochemistry, Biocenter, Goethe University Frankfurt, Max-von-Laue-Str. 9, 60438 Frankfurt a.M., Germany;

"Institute for Biophysical Chemistry and Centre for Biomolecular Magnetic Resonance, Goethe University Frankfurt, Max-von-Laue-Str. 9, 60438 Frankfurt a.M., Germany;

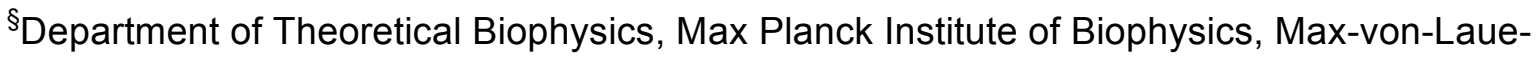

Str. 3, 60438 Frankfurt a.M., Germany.

*For correspondence:

glaubitz@em.uni-frankfurt.de (C. G.) and tampe@em.uni-frankfurt.de (R. T.)

$\left(^{\ddagger}\right)$ These authors contributed equally 
Table S1. Chemical shift assignment of KRYQNSTVY bound to TAP.

$\delta$ [ppm] (line width [Hz])

\begin{tabular}{|c|c|c|c|c|c|c|c|c|c|c|c|}
\hline residue & NA & $\mathrm{CO}$ & $\mathrm{CA}$ & CB & CG & $C D$ & CE & $C Z$ & ND/NZ & NE & NH \\
\hline Lys1 & $\begin{array}{c}39.2 \\
(310) \\
43.4 \\
(260)\end{array}$ & $\begin{array}{l}172.8 \\
(570)\end{array}$ & $\begin{array}{r}55.5 \\
(400)\end{array}$ & n.d. & n.d. & $\begin{array}{r}29.8 \\
\text { (n.d.) }\end{array}$ & $\begin{array}{r}42.6 \\
(300)\end{array}$ & - & $\begin{array}{r}33.5 \\
(250)\end{array}$ & & \\
\hline Arg2 & $\begin{array}{r}119.0 \\
(580)\end{array}$ & $\begin{array}{l}175.8 \\
(460)\end{array}$ & $\begin{array}{r}55.2 \\
(520)\end{array}$ & n.d. & n.d. & $\begin{array}{r}43.4 \\
(370)\end{array}$ & - & $\begin{array}{l}159.1 \\
(250)\end{array}$ & _ & $\begin{array}{r}84.6 \\
(350)\end{array}$ & $\begin{array}{r}73.3 \\
(400)\end{array}$ \\
\hline Tyr3 & $\begin{array}{c}120.3 \\
(800)\end{array}$ & $\begin{array}{l}175.5 \\
(590)\end{array}$ & $\begin{array}{r}57.4 \\
(670)\end{array}$ & $\begin{array}{r}38.3 \\
(490)\end{array}$ & n.d. & $\begin{array}{l}133.8 \\
(440)\end{array}$ & $\begin{array}{l}118.0 \\
(280)\end{array}$ & $\begin{array}{l}158.0 \\
(430) \\
159.3 \\
(430)\end{array}$ & & & \\
\hline GIn4 & $\begin{array}{r}120.3 \\
(800)\end{array}$ & $\begin{array}{l}176.4 \\
(590)\end{array}$ & $\begin{array}{r}56.0 \\
(670)\end{array}$ & n.d. & $\begin{array}{r}33.9 \\
(610)\end{array}$ & $\begin{array}{l}180.2 \\
(470)\end{array}$ & - & - & - & $\begin{array}{l}116.1 \\
(800)\end{array}$ & \\
\hline Asn5 & $\begin{array}{c}116.5^{*} \\
(680)\end{array}$ & $\begin{array}{l}176.0 \\
(540)\end{array}$ & $\begin{array}{r}52.5 \\
(520)\end{array}$ & $\begin{array}{r}39.2 \\
(340)\end{array}$ & $\begin{array}{l}177.2 \\
(490)\end{array}$ & - & - & - & n.d. & & \\
\hline Ser6 & $\begin{array}{c}112.1^{*} \\
(440)\end{array}$ & $\begin{array}{l}174.5 \\
(400)\end{array}$ & $\begin{array}{r}57.8 \\
(310)\end{array}$ & n.d. & & & & & & & \\
\hline Thr7 & $\begin{array}{c}125.0^{*} \\
(460)\end{array}$ & $\begin{array}{l}175.2 \\
(540)\end{array}$ & $\begin{array}{r}61.2 \\
(570)\end{array}$ & $\begin{array}{r}69.1 \\
(310)\end{array}$ & $\begin{array}{r}22.9 \\
(420)\end{array}$ & & & & & & \\
\hline Val8 & $\begin{array}{c}112.1^{*} \\
(460)\end{array}$ & $\begin{array}{l}175.2 \\
\text { (540) }\end{array}$ & $\begin{array}{r}61.2 \\
(570)\end{array}$ & $\begin{array}{r}33.7 \\
(280)\end{array}$ & n.d. & & & & & & \\
\hline Tyr9 & $\begin{array}{c}129.5 \\
(600) \\
122.4 \\
(600)\end{array}$ & $\begin{array}{l}181.4 \\
(580)\end{array}$ & $\begin{array}{r}58.6 \\
(480)\end{array}$ & $\begin{array}{r}38.6 \\
(440)\end{array}$ & n.d. & $\begin{array}{l}133.8 \\
(410)\end{array}$ & $\begin{array}{l}118.2 \\
(630)\end{array}$ & $\begin{array}{c}157.2 \\
(230) \\
158.5 \\
(180)\end{array}$ & & & \\
\hline
\end{tabular}

n.d. not detectable or ambiguous due to overlap; "values represent peak maxima and were excluded from TALOS-N calculation due to broad distribution, respective line widths are shown without commitment. 
Table S2. Chemical shift assignment of KRYQNSTVY in GDN buffer.

\begin{tabular}{|c|c|c|c|c|c|c|c|c|c|c|c|}
\hline \multirow[b]{2}{*}{ residue } & \multicolumn{11}{|c|}{$\delta$ [ppm] (line width [Hz]) } \\
\hline & NA & co & CA & CB & CG & CD & CE & CZ & ND/NZ & NE & NH \\
\hline Lys1 & $\begin{array}{c}38.1 \\
(440)\end{array}$ & $\begin{array}{l}172.9 \\
(430)\end{array}$ & $\begin{array}{r}55.3 \\
(330)\end{array}$ & $\begin{array}{r}33.8 \\
\text { (n.d.) }\end{array}$ & n.d. & $\begin{array}{r}30.0 \\
\text { (n.d.) }\end{array}$ & $\begin{array}{r}42.2 \\
(320)\end{array}$ & - & $\begin{array}{r}32.4 \\
(320)\end{array}$ & & \\
\hline Arg2 & $\begin{array}{r}119.3 \\
(590)\end{array}$ & $\begin{array}{l}175.9 \\
(570)\end{array}$ & $\begin{array}{r}55.0 \\
(580)\end{array}$ & $\begin{array}{r}31.6 \\
(590)\end{array}$ & $\begin{array}{r}28.3 \\
\text { (n.d.) }\end{array}$ & $\begin{array}{r}43.5 \\
(400)\end{array}$ & - & $\begin{array}{l}159.1 \\
(290)\end{array}$ & - & $\begin{array}{r}84.8 \\
(330)\end{array}$ & $\begin{array}{r}73.5 \\
(330)\end{array}$ \\
\hline Tyr3 & $\begin{array}{r}118.5 \\
(940)\end{array}$ & $\begin{array}{l}175.5 \\
(570)\end{array}$ & $\begin{array}{r}57.4 \\
(720)\end{array}$ & $\begin{array}{r}38.4 \\
(440)\end{array}$ & n.d. & $\begin{array}{l}134.2 \\
(550)\end{array}$ & $\begin{array}{l}117.8 \\
(510)\end{array}$ & $\begin{array}{l}158.4 \\
(330)\end{array}$ & & & \\
\hline Gln4 & $\begin{array}{c}118.5 \\
(940)\end{array}$ & $\begin{array}{l}176.2 \\
(570)\end{array}$ & $\begin{array}{r}55.9 \\
(720)\end{array}$ & n.d. & $\begin{array}{r}33.6 \\
(520)\end{array}$ & $\begin{array}{l}180.9 \\
(420)\end{array}$ & - & - & - & $\begin{array}{l}116.3 \\
(700)\end{array}$ & \\
\hline Asn5 & $\begin{array}{c}117.4^{*} \\
(670)\end{array}$ & $\begin{array}{l}176.0 \\
(530)\end{array}$ & $\begin{array}{r}52.3 \\
(430)\end{array}$ & $\begin{array}{r}38.1 \\
(500)\end{array}$ & $\begin{array}{l}177.2 \\
(440)\end{array}$ & - & - & - & n.d. & & \\
\hline Ser6 & $\begin{array}{c}114.3^{*} \\
(680)\end{array}$ & $\begin{array}{l}174.5 \\
(530)\end{array}$ & $\begin{array}{r}57.7 \\
(580)\end{array}$ & $\begin{array}{r}63.4 \\
(350)\end{array}$ & & & & & & & \\
\hline Thr7 & $\begin{array}{c}121.5^{*} \\
(630)\end{array}$ & $\begin{array}{l}174.7 \\
(470)\end{array}$ & $\begin{array}{r}60.7 \\
(500)\end{array}$ & $\begin{array}{r}69.0 \\
(550)\end{array}$ & $\begin{array}{r}22.2 \\
(400)\end{array}$ & & & & & & \\
\hline Val8 & $\begin{array}{c}115.2^{*} \\
(810)\end{array}$ & $\begin{array}{l}174.7 \\
(470)\end{array}$ & $\begin{array}{r}60.7 \\
(500)\end{array}$ & $\begin{array}{r}32.0 \\
(420)\end{array}$ & $\begin{array}{r}21.3 \\
(470)\end{array}$ & & & & & & \\
\hline Tyr9 & $\begin{array}{r}121.8 \\
(820)\end{array}$ & $\begin{array}{l}181.4 \\
(520)\end{array}$ & $\begin{array}{r}58.6 \\
(580)\end{array}$ & $\begin{array}{r}38.8 \\
(520)\end{array}$ & n.d. & $\begin{array}{l}133.8 \\
(560)\end{array}$ & $\begin{array}{l}118.4 \\
(460)\end{array}$ & $\begin{array}{l}158.1 \\
(390)\end{array}$ & & & \\
\hline
\end{tabular}

n.d. not detectable or ambiguous due to overlap; "values represent peak maxima and were excluded from TALOS-N calculation due to broad distribution, respective line widths are shown without commitment. 
Table S3. Torsion angle restraints of KRYQNSTVY bound to TAP derived from TALOS-N.

\begin{tabular}{|c|c|c|c|c|c|c|c|c|c|c|c|c|}
\hline \multirow{2}{*}{$\begin{array}{c}\text { residue } \\
\text { Lys1 }\end{array}$} & \multicolumn{3}{|c|}{$\varphi_{\min } \ldots \varphi_{\max }$} & \multicolumn{3}{|c|}{$\varphi_{\text {mean }} \pm \mathrm{SD}$} & \multicolumn{3}{|c|}{$\psi_{\min } \ldots \psi_{\max }$} & \multicolumn{3}{|c|}{$\psi_{\text {mean }} \pm S D$} \\
\hline & & - & & & - & & & - & & & - & \\
\hline Arg2 & -77.2 & $\ldots$ & -60.3 & -68.8 & \pm & 8.5 & 133.8 & $\ldots$ & 156.4 & 145.1 & \pm & 11.3 \\
\hline Tyr3 & -78.5 & $\ldots$ & -60.9 & -69.7 & \pm & 8.8 & 136.1 & $\ldots$ & 151.1 & 143.6 & \pm & 7.5 \\
\hline GIn4 & -79.1 & $\ldots$ & -56.8 & -68.0 & \pm & 11.2 & 136.0 & $\ldots$ & 167.4 & 151.7 & \pm & 15.7 \\
\hline Asn5 & -82.9 & $\ldots$ & -62.3 & -72.6 & \pm & 10.3 & 133.9 & $\ldots$ & 156.2 & 145.1 & \pm & 11.2 \\
\hline Ser6 & -119.1 & $\ldots$ & -79.2 & -99.2 & \pm & 20.0 & 129.1 & $\ldots$ & 151.5 & 140.3 & \pm & 11.2 \\
\hline Thr7 & -112.2 & $\ldots$ & -90.2 & -101.2 & \pm & 11.0 & 121.3 & $\ldots$ & 137.0 & 129.2 & \pm & 7.9 \\
\hline Val8 & -119.4 & $\ldots$ & -101.4 & -110.4 & \pm & 9.0 & 120.6 & $\ldots$ & 134.2 & 127.4 & \pm & 6.8 \\
\hline Tyr9 & & - & & & - & & & - & & & - & \\
\hline
\end{tabular}

Table S4. Torsion angle restraints of KRYQNSTVY in GDN buffer derived from TALOS-N.

\begin{tabular}{|c|c|c|c|c|c|c|c|c|c|c|c|c|}
\hline \multirow{2}{*}{$\begin{array}{c}\text { residue } \\
\text { Lys1 }\end{array}$} & \multicolumn{3}{|c|}{$\varphi_{\min } \ldots \varphi_{\max }$} & \multicolumn{3}{|c|}{$\varphi_{\text {mean }} \pm \mathrm{SD}$} & \multicolumn{3}{|c|}{$\psi_{\min } \ldots \psi_{\max }$} & \multicolumn{3}{|c|}{$\psi_{\text {mean }} \pm S D$} \\
\hline & & - & & & - & & & - & & & - & \\
\hline Arg2 & -102.6 & $\ldots$ & -64.3 & -83.5 & \pm & 19.2 & 142.1 & $\ldots$ & 160.5 & 151.3 & \pm & 9.2 \\
\hline Tyr3 & -83.8 & $\ldots$ & -62.5 & -73.2 & \pm & 10.6 & 135.3 & $\ldots$ & 152.1 & 143.7 & \pm & 8.4 \\
\hline GIn4 & -98.6 & $\ldots$ & -54.6 & -76.6 & \pm & 22.0 & 141.4 & $\ldots$ & 171.8 & 156.6 & \pm & 15.2 \\
\hline Asn5 & -78.9 & $\cdots$ & -60.5 & -69.7 & \pm & 9.2 & 133.4 & $\ldots$ & 157.4 & 145.4 & \pm & 12.0 \\
\hline Ser6 & -99.9 & $\ldots$ & -76.8 & -88.4 & \pm & 11.6 & 124.5 & $\ldots$ & 149.1 & 136.8 & \pm & 12.3 \\
\hline Thr7 & -116.5 & $\ldots$ & -97.9 & -107.2 & \pm & 9.3 & 122.7 & $\ldots$ & 135.3 & 129.0 & \pm & 6.3 \\
\hline Val8 & -114.3 & $\cdots$ & -101.8 & -108.1 & \pm & 6.3 & 116.0 & $\ldots$ & 133.5 & 124.8 & \pm & 8.8 \\
\hline Tyr9 & & - & & & - & & & - & & & - & \\
\hline
\end{tabular}


Table S5. Comparison of residues within the substrate-binding site revealed by peptide docking (4 A cut-off for interactions) and previous studies.

docking

N-terminal part

\begin{tabular}{|c|c|c|c|}
\hline & $\begin{array}{c}\mathrm{S} 293^{\text {TAP1 }}, \mathrm{D} 297^{\text {TAP1 }} \\
\text { E301 }\end{array}$ & $\mathrm{S} 296^{\text {TAP1 }}$ & 2 \\
\hline & $\mathrm{R} 467^{\mathrm{TAP} 1}$ & $\mathrm{E} 459^{\text {TAP1 }}$ & 2 \\
\hline & $\mathrm{R} 373^{\text {TAP2 }}$ & $\mathrm{A} 374^{\mathrm{TAP} 2}, \mathrm{R} 380^{\mathrm{TAP2}}$ & $3-5$ \\
\hline & $Y 428^{\text {TAP2 }}$ & - & - \\
\hline \multirow[t]{4}{*}{ C-terminal part } & $\begin{array}{c}\text { W413 }{ }^{\text {TAP1 }}, S 416^{\text {TAP1 } 1} \\
K^{\prime} 423^{\text {TAP1 }}\end{array}$ & Y408 ${ }^{\text {TAP1 }}$ & 2 \\
\hline & $\mathrm{S} 203^{\text {TAP2 }}, \mathrm{R} 210^{\text {TAP2 }}$ & $\mathrm{C} 213^{\text {TAP2 }}, \mathrm{T}_{217^{\mathrm{TAP} 2}}, \mathrm{M} 218^{\mathrm{TAP2}}$ & $2,4-6$ \\
\hline & $\mathrm{N} 267^{\text {TAP2 }}$ & $\mathrm{N} 262^{\text {TAP2 }}, \mathrm{P} 265^{\text {TAP2 }}, \mathrm{L}^{266^{\text {TAP2 }}}$ & 5 \\
\hline & $\begin{array}{c}\mathrm{E} 166^{\mathrm{TAP2}}, \mathrm{K} 277^{\mathrm{TAP2} 2} \\
\mathrm{~S} 421^{\text {TAP2 }}\end{array}$ & - & - \\
\hline
\end{tabular}

reference

$\mathrm{G}^{282^{\text {TAP1 }}}$, I284 ${ }^{\text {TAP1 }}$,
R2 $^{\text {TAP1 }}, 7^{\text {TA2 }} 288^{\text {TAP1 }}$

E290 ${ }^{\text {TAP }}$

$$
\text { G282 } 2^{\text {TAP1 }}, 1284^{\text {TAP1 }} \text {, }
$$

1

Residue numbers refer to human TAP

Table S6. Representative acquisition and processing parameters for ssNMR experiments.

\begin{tabular}{|c|c|c|}
\hline & DQ-SQ $\left({ }^{13} \mathrm{C}-{ }^{13} \mathrm{C}\right)$ & TEDOR $\left({ }^{15} \mathrm{~N}-{ }^{13} \mathrm{C}\right)$ \\
\hline F2 digital resolution [Hz] & 30 & 30 \\
\hline F1 digital resolution* [Hz] & 1167 & $62-83$ \\
\hline interscan delay [s] & 3 & 3 \\
\hline number of scans & $512-2048$ & $2048-4096$ \\
\hline F2 window function ${ }^{\star *}$ & exponential & exponential \\
\hline F1 window function** & $\begin{array}{l}\text { exponential or squared } \\
\text { Gaussian }\end{array}$ & $\begin{array}{l}\text { exponential or squared } \\
\text { Gaussian }\end{array}$ \\
\hline
\end{tabular}

${ }^{*} \mathrm{~F} 1$ dimensions in ${ }^{13} \mathrm{C}-{ }^{13} \mathrm{C} \mathrm{DQ}-\mathrm{SQ}$ spectra were not used to obtain ${ }^{13} \mathrm{C}$ chemical shifts.

** Line broadening factors are optimized in a range of $50-150 \mathrm{~Hz}$ according to sensitivity, spectral resolution, and signal overlapping. 


\section{Figure S1}

(a)

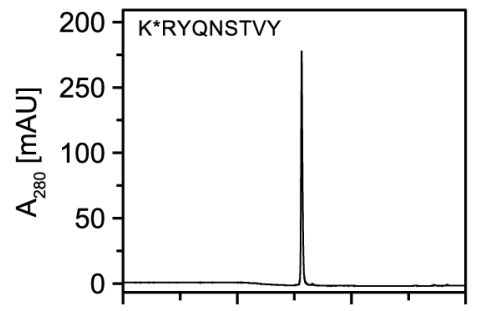

(b)

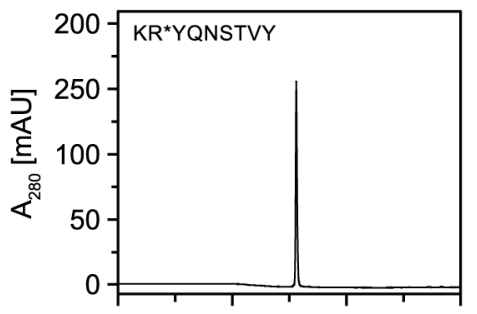

(c)

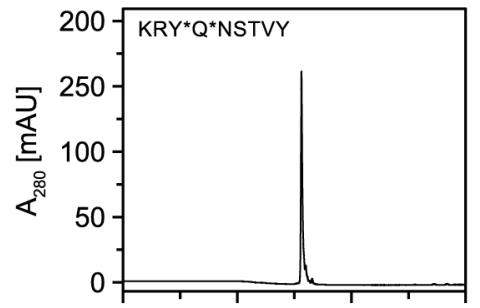

(d)

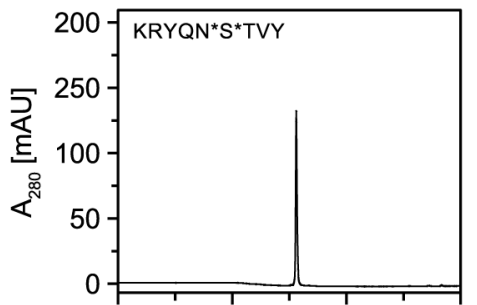

(e)

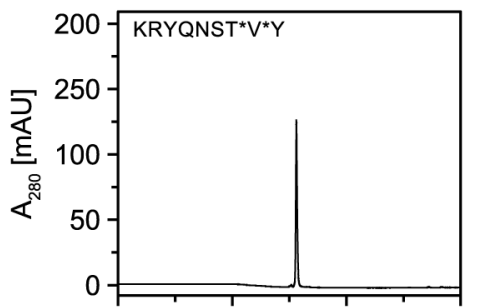

(f)

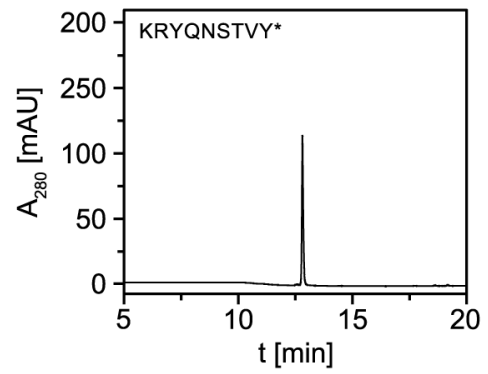

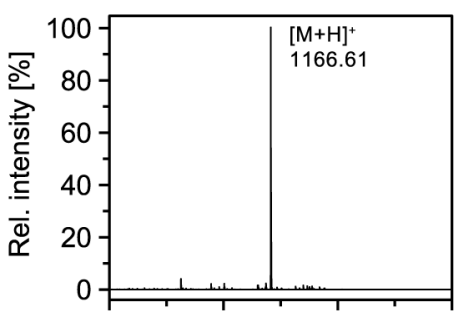
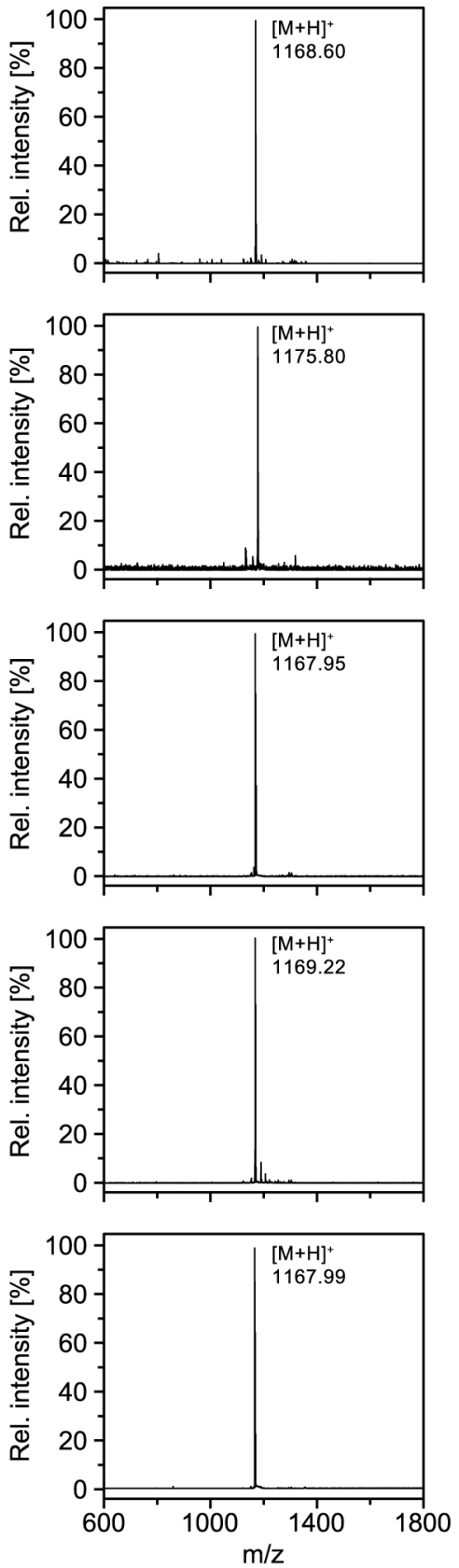

Figure S1. Purity and identity of isotope-labeled peptides. The purity and identity of

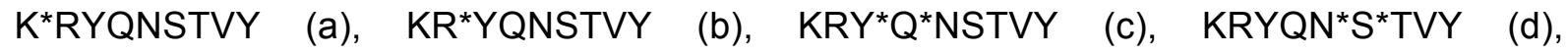
$\mathrm{KRYQNST}^{*} \mathrm{~V}^{*} \mathrm{Y}(\mathrm{e})$, and KRYQNSTVY* (f) were verified by RP-C ${ }_{18}$-HPLC (left) and MALDITOF mass spectrometry (right), respectively. Chromatographic analysis was performed with a linear gradient from $5 \%$ to $100 \%(\mathrm{v} / \mathrm{v})$ acetonitrile supplemented with $0.1 \%(\mathrm{v} / \mathrm{v})$ trifluoroacetic acid. Asterisks indicate uniformly ${ }^{15} \mathrm{~N},{ }^{13} \mathrm{C}$-labeled amino acids. 


\section{Figure S2}
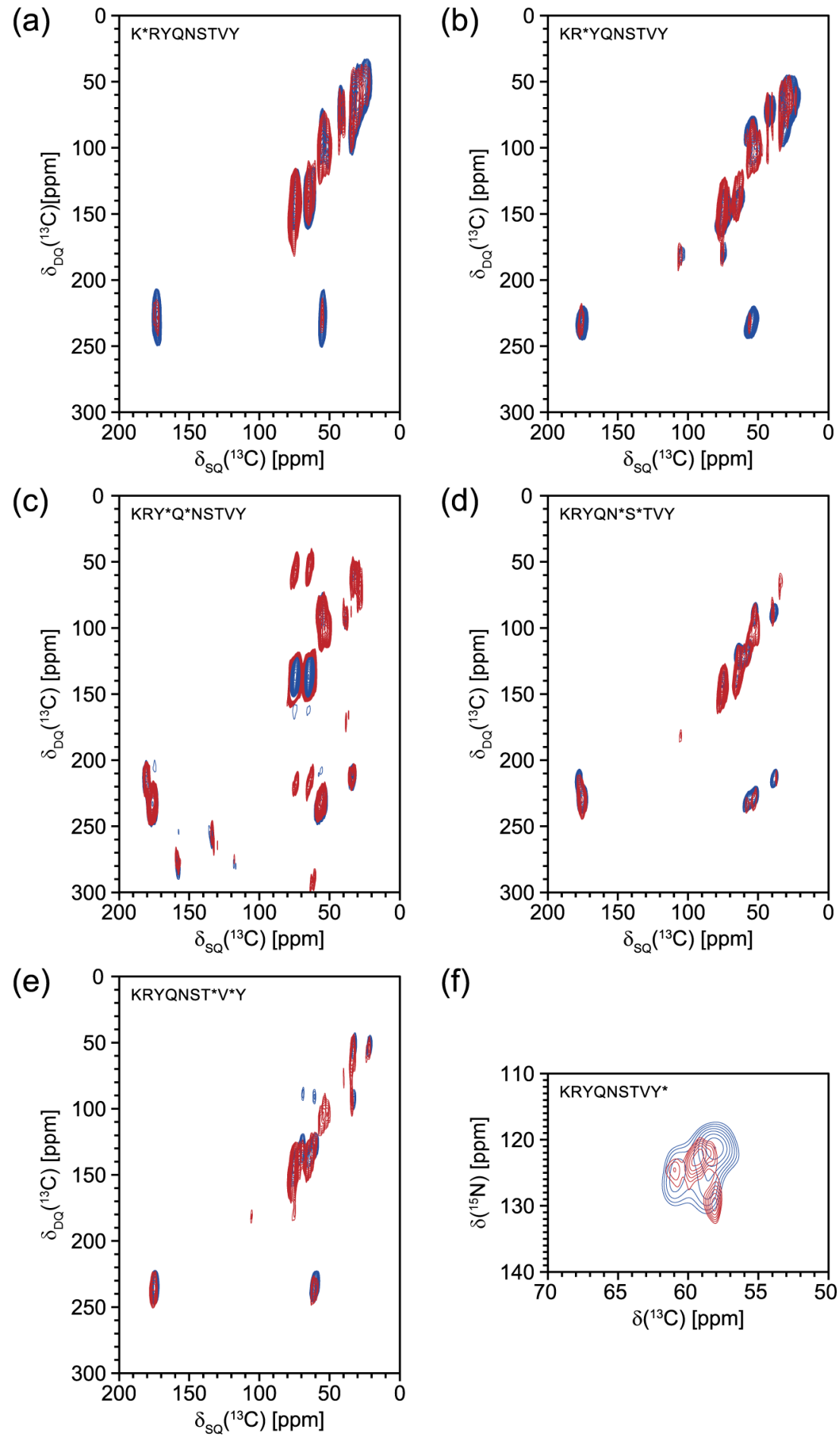

(f)

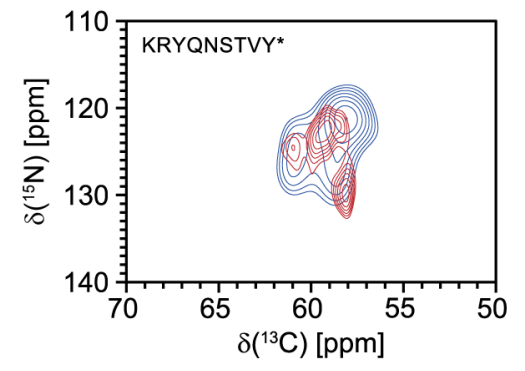

Figure S2. DQ-SQ and TEDOR ssNMR spectra of peptides in complex with TAP (red) and in GDN buffer (blue). (a) K*RYQNSTVY, (b) KR*YQNSTVY, (c) KRY* ${ }^{*} N S T V Y$, (d) KRYQN* ${ }^{*} T V Y$, (e) KRYQNST* ${ }^{*} Y$, and (f) KRYQNSTVY*. Asterisks indicate uniformly ${ }^{15} \mathrm{~N},{ }^{13} \mathrm{C}$-labeled amino acids. For further details see Materials and Methods. 


\section{Figure S3}
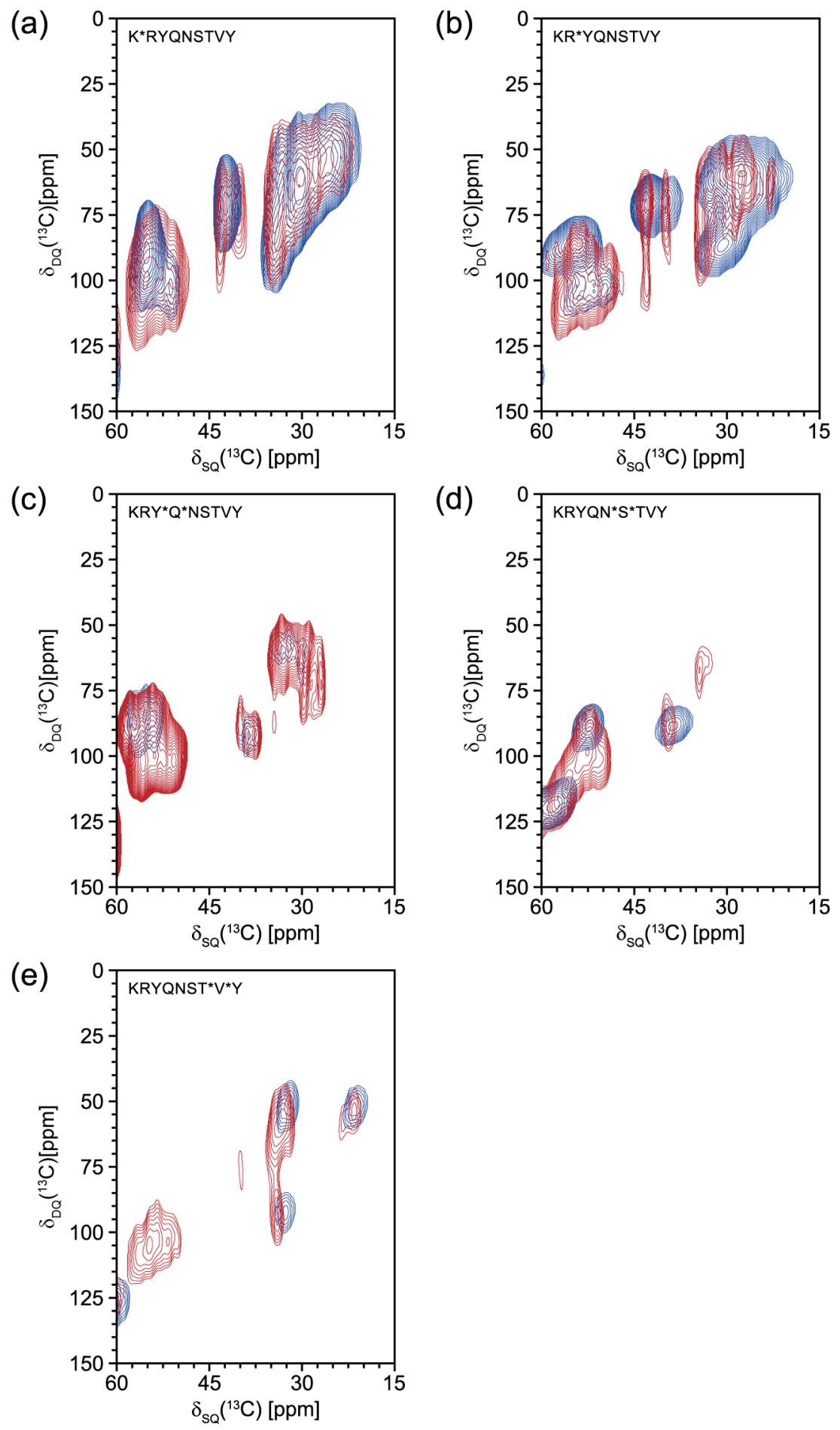

Figure S3. Aliphatic regions of DQ-SQ and TEDOR sSNMR spectra of peptides in complex with TAP (red) and in GDN buffer (blue). (a) K*RYQNSTVY, (b) KR*YQNSTVY, (c) $K R{ }^{*} Q^{*} N S T V Y$, (d) KRYQN*S*TVY, and (e) KRYQNST ${ }^{*}{ }^{*} Y$. Asterisks indicate uniformly ${ }^{15} \mathrm{~N},{ }^{13} \mathrm{C}$-labeled amino acids. For further details see Materials and Methods. 


\section{Figure S4}

(a)

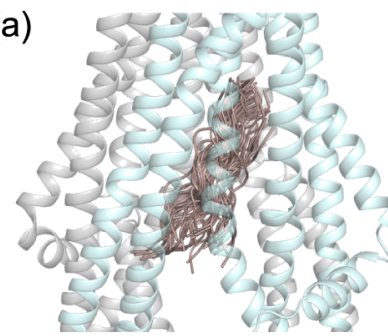

(c)

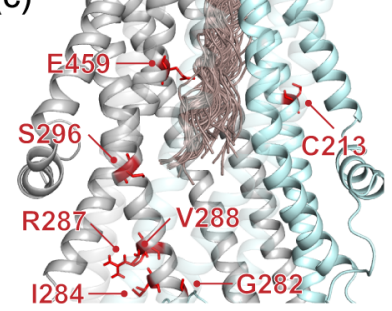

(b)

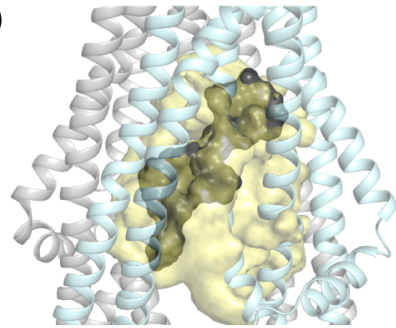

(d)

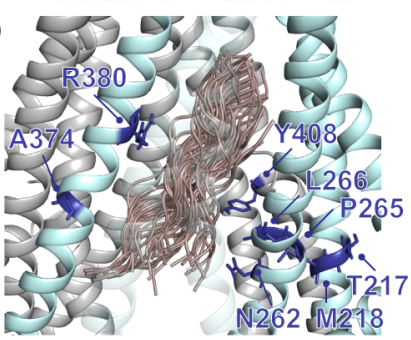

Figure S4. (a) Bundle of docked peptides (62 poses; raspberry). TAP1 and TAP2 are colored grey and cyan, respectively. (b) A large cavity (yellow) surrounds the TAP-bound peptide (dark grey) and provides additional space for fluorophores, chemical proteases, branched polylysine side chains, or extra long peptides. (c and d) Residues contributing to substrate-binding/translocation of TAP revealed by (c) cross-linking (red) and (d) mutational analyses (blue) from previous studies (see Table S5). 


\section{References}

(1) Herget, M.; Oancea, G.; Schrodt, S.; Karas, M.; Tampé, R.; Abele, R. J Biol Chem 2007, 282, 3871.

(2) Geng, J.; Pogozheva, I. D.; Mosberg, H. I.; Raghavan, M. J Immunol 2015, 195, 3436.

(3) Armandola, E. A.; Momburg, F.; Nijenhuis, M.; Bulbuc, N.; Früh, K.; Hämmerling, G. J. Eur J Immunol 1996, 26, 1748.

(4) Momburg, F.; Armandola, E. A.; Post, M.; Hämmerling, G. J. J Immunol 1996, 156, 1756.

(5) Deverson, E. V.; Leong, L.; Seelig, A.; Coadwell, W. J.; Tredgett, E. M.; Butcher, G. W.; Howard, J. C. J Immunol 1998, 160, 2767.

(6) Baldauf, C.; Schrodt, S.; Herget, M.; Koch, J.; Tampé, R. Proc Natl Acad Sci U S A 2010, 107, 9135. 